\title{
TABLE
}

\section{PAR ORDRE ALPHABÉTIQUE DES NOMS D'AUTEURS}

\section{IDES PUBLICATIONS}

\author{
ANALYSÉES DANS LA REVUE BIBLIOGRAPHIQUE.
}

(TOME CINQUUÈME.)

\begin{abstract}
N. B. - Cette table ne contient que les titres des ouvrages analysés et les noms de leurs auteurs. Tous les noms de plantes dont les descriptions ou les diagnoses se trouvent reproduites dans la Revue bibliographique, ainsi que les articles nécrologiques, etc., doivent être cherchés dans la table générale qui précède celle-ci. - Cette observation s'applique également aux tables des tomes I à IV, où elle a été omise.
\end{abstract}

Axonyme. Vitalité des Fougères (par W. P.), Camara-Leme (J. da). Études sur les Om376. bellifères vénéneuses, 197.

Ascaensos (P.). Remarques sur les espèces Carter (H.-J.). Sur les caractères spécifi. d'Anacyclus qui fournissent la racine ques, la fécondation et le développement Bertram ou racine de Pyrèthre, 134. anormal des OEdogonium, 131.

CARUel (Th.). Illustratio in hortum siccum Andrece Cosalpini, 306.

Baillon (H.). Étude générale du groupe CAspary (R.). Les Hydrillées (Anacharidées des Euphorbiacées, 776 .

Balansa (B.), voy. Boissier. Endl.), 296.

Césalpin (A.), voy. Caruel.

BatLy (V.). Notice sur la vie et les travaux de Villar, 309.

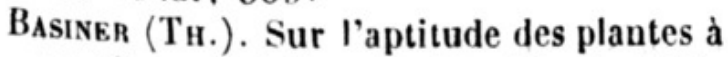
se plier aux influences des climats, 781 .

Benthas (G.). Synopsis du genre Clitoria, 69. - Note sur Ins principes de la nomenclature des genres en botanique, 75 .

Berkeley (M.-J.). Sur quelques Sphéries entomogènes, 68 .

BleEkrod. Notice sur la gutta-percha de Surinam, 74.

BöCKeler. Choricarpha, genre nouv. et remarquable de Cypéracées, 233.

Chatrs (Ad.). Anatomie des plantes aériennes de l'ordre des Orchidées $\left(2^{\mathbf{e}}\right.$ mém.), 645.

Chorsy (J.-D.). Planter javanica nec non ex insuiis finitimis et eliam e Japonia quadam oriunda, in Zollingeri catalogo elaboratee, 661 .

Clos (D.). Origine des Champignons. La Truffe et sa culture, 199. - Pourret et son histoire des Cistes, 291.

Corenwinder (B.). Recherches sur l'assimilation du carbone par les feuilles des végétaux, 764 .

Boissier (E.) et Balansa. Description du Cramer (C.). Sur la manière dont se comgenre Thurya, 234.

Волномме (J.). Note sur quelques Algues d'eau douce, 663.

Bunorden. Obs. sur la formation du Spermœedia Clavus ou Seigle ergoté, 290.

Boreau (A.). Flore du centre de la France Currex (Fr.). Sur une espèce de Pilobolus, et du bassin de la Loire ( $3^{\mathrm{e}}$ édit. ), 60 . porte l'oryde de cuivre ammoniacal avec la membrane des cellules, avec la fécule, l'inuline, le nucléus cellulaire et l'utricule primordiale, 373. - Voy. Nægeli.

66.

Botanical Magazine (cahier de mars 1858), 241 ; (c. d'avril), 242 ; (c. de mai et de juin), 670 .

Boussingault. Alimentation des plantes. De Bary (A.). Sur les Orchis militaris, SiInfluence du phosphate de chaux des mia, fusca et leurs hybrides, 637. engrais sur la production végétale, 58. DeBEx (M.-H.), voy. Ettingshausen. 
Decaisse (J.). Le Jardin fruitier du Muséum (t. I), 397.

Des Mouliss (Ch.). Sur les Chrysanthèmes d'automne de nos jardins, et sur quelques plantes qui leur sont congénères, 570.

Duval-Jouve (J.). Études sur le pétiole des Fougè res, 566 .

Etrisgshausen (C. d') et Debey. Les Thallophytes fossiles de la formation crétacée d'Aix-la-Chapelle, 304 .

Fenzl (Éd.), voy. Wulfen.

Flora (journal). Articles originaux, 79.

Fourcy (E. de). Vade-mecum des herborisations parisiennes, 768 .

Gasparkini (G.). Recherches sur la naturo les suçoirs et sur les excrétions des racines, 648 .

Geısıtz (H.-B.). Les plantes caractéristiques du grès rouge et du zechstein ou de la formation permienne en Saxe, 665 .

Gocppert. Sur la forêt pétrifiée de Radowenz (Bohême) et sur la fossilisation en général, 111.

Graf (P.-R.), voy. Wulfen.

Gray (Asa). Comment les plantes poussent, simple introduction à la botanique structurale, avec une flore populaire, 655 .

Gray (J. -E.). Note sur l'Anemone nemorosa purpurea, 136.

Groesland (J.). Quelques mots sur la formation des hybrides dans le genre $\mathrm{Kg}$ ilops, 655 .

Guembel. (W.). Sur la pourriture ver̂te du bois, 375 .

Hamburger Garten- und Blumenzeitung (journal), $\mathbf{5}^{\mathrm{e}}$ cahier de $\mathbf{1 8 5 8}, 395$.

Hanstein (H.). Sur les rapports des plantes avec la nature du sol, 72. - Sur l'absorption de l'azote par les plantes au moyen des acides de l'humus, 227 .

Hesfrey (A.). Sur les rapports du raphé avec les téguments de l'ovule végétal, 178. - Note sur le contenu cellulaire des Clostéries, 285.

HetfFel (J.). Diagnoses des plantes nouvelles on litigieuses du Banat, 295

Hildebraxd (F.-A.-G.). De caulibus Begoniacearum, imprimis is qui vasorum fasciculis in parenchymate medullari dispersis sunt proditi, 760 .

Hofmister (W.). Sur l'ascension de la séve dans les plantes, 377 .

Holle (de). Sur le Champignon des Pommes de terre, 228 .

Hooker (J.-D.) et Thomson. Pracursores ad floram indicam, 63.

Inmısce (Th.). Notes botaniques (Utricularia minor. Crocus. Germination du Bunium creticum. Poires monstrueuses. Chaume de Seigle extraordinairement ramifié), 128-131. - Pour I'histoire naturelle du Melittis Melissophyllum, 375 .

J EGER. Sapins remarquables, 784 .

JARDIN (Ed.). Essai sur I'histoire naturelle de l'archipel de Mendana ou des Marquises, 771.

Karsten (H.). Les écorces médicinales de quinquina de la Nouvelle-Grenade, 666 .

Kinschleger (Fr.). Flore d'Alsace (t. III, $1^{\text {re }}$ part. Végétation rhénano-vosgienne), 571 .

Korschy (Th.). La végétation et le canal de l'isthme de Suez, 193.

Lacroix (l'abbé S. de). Nouy. faits constatés relativement a l'histoire de la botanique et à la distribution géograph. des plantes de la Vienne, 184.

Langethal. (Chr.-Ed.). Descript. des plantes de l'Allemagne d'après leurs fam. naturelles et leur importance pour l'agriculture, 382.

Lecoe (H.). Études sur la géographie botanique de l'Europe, 236.

LindeEY. Liste d'Orchidées récoltées par M. Wright dans la portion orientale de Cuba, 189.

Marquart. Sur les qualités du Sorbus aucuparia, 196.

Martiv (B.). Note sur le Scleranthus uncinatus Schur, 636.

Mathiev (A.). Flore forestière, 573.

Michel (A.P.), voy. Rondot.

Miers (J.). Sur la nature et l'origine des téguments externes des graines, 126 . Sur les Canellacées, 140. - Nouv. obs. sur la nature et l'origine des téguments 
externes des graines, 177. - Sur les Wintéracées, 387.

MiLde (J.). Sur ie Botrychium lanceolatum Angstr., 234.

Moнl (H. de). Examen du tissu végétal à l'aide de la lumière polarisée, 49.

Morren (Éd.). Dissertation sur les feuilles vertes et colorées, envisagées spécialement au point de vue des rapports de la chlorophylle et de l'érythrophylle, 173.

Mugleer (J.). Monographie de la famille des Résédacées, 70.

Nageli (Ch.) et Cramer. Recherches de physiologie végétale $\left(4^{\mathrm{e}}\right.$ cahier, par M. Cramer : Céramiées), 287.

OLIver (D.). Note relative à certains appendices glanduleux des feuilles des rosettes automnales de l'Epilobium montanum, 60.

PARISOT (L.). Notice sur la flore des env. de Belfort, 567 .

Parlatore (Ph.). Deux nouv. genres de plantes monocotylédones (Ruminia et Barlia), 775 .

Persoz (J.), voy. Rondot.

Philippi (R.-A.). Plantarum novarum chilensium centuria prima, 186; altera, 187; tertia, 188. - Statistique de la flore du Chili, 390 .

Planchon (J.-E.). Hortus donatensis, 190. - Sur le parasitisme de l'Osyris alba, 289.

Plantes nouv. ou rares décrites dans les publications horticoles, $241,395,670$.

Pokorny (Al.). Formes de l'Equiselum arvense et des espèces du groupe des $E$. hiemalia, 137.

Povrret, voy. Clos.

$R_{\text {Atchinsky }}$ (S.). Notice sur quelques mouvements opérés par les plantes sous l'influence de la lumière, 762 .

REgel. (Éd.). Rapport sur les essais de fécondation des Plalycentrum rubrovenium et xanthinum l'un par l'autre, 633. - Les espèces d'Agave du jardin botanique de Saint-Pétersbourg, 662.

Reichardi (H.-W.). Notes sur les bourgeons adventifs hypocotylés et sur les pousses des racines ou les drageons dans les Dicotylédons herbacés, 180. - Sur la distrib. des faisceaux vasculaires dans la tige et le pétiole des Fougères, 283.

Reichenbach (L. et H.-G.). Icones flora germanica et helveticis $\mathrm{t}$. XVIII, dec. 7-8', 233; (dec. 9-15), 29 เ.

Reıssch (P.-Fr.). Sur quelques anomalies morphologiques : Salix cinerea, 133; Equisetum Telmateia, 134.

Rochleder (Fr.). Introduct. à l'analyse des plantes et des parties des plantes, 143 . - Chimie et physiologie végétales, 379 . Roxdor (N.). Notice du vert de Chine et de la teinture en vert chez les Chinois, suivie d'une Étude des propriétés chimiques et tinctoriales du Lo-Kao (par M. Persoz) et de Recherches sur la matière colorante des Nerpruns indigènes (par M. Michel), 244.

RosBach. Sur quelques variations de forme de l'Orchis fusca Jacq., 185.

Roumeguére (C.). Note sur une nouv. esp. de Lichen (Usnea saxicola), 138.

Sacrs (J.). Sur les organes moteurs et sur les mouvements périodiques des feuilles dans les Phaseolus et les Oxalis, 222.

Schacer (H.). Nouv. recherches sur la fécondation dans le Gladiolus segetum, $56,226$.

Schlechtendal (D. -F.-L. de). Note relative à I'histoire des Sparganium de I'Allemagne, 381 .

SCHWEIzER (Éd.). Lioxyde de cuivre ammoniacal dissolvant de la fibre végétale, 373.

Sermans (B.). Sur le Palmier de Tombouctou, 65 .

Soubeırax (Léon). Essai sur la matière organisée des sources sulfureuses des Pyrénées, 300.

\section{Thomsos (T), voy. Hooker.}

Thuemex-G Gefendorf ( $F$. de $)$. Enumération systémat. des esp. d'Hieracium de l'Allemagne, de l'empire d'Autriche et de la Suisse, 136. - Distribut. géographique du genre Cyperus, 303.

Todaro (Aug. Nouv. genres et nouv. esp. cultivés dans le jardin botanique de Palerme, 659.

Trautvetter (E.-R. de). Sur le Belula davurica Pall., 770.

Trécul (A.). De la présence du latex dans les vaisseaux spiraux, réticulés, rayés et ponctués, et de la circulation dans les plantes, 34. - Sur des cristaur orgaganisés et vivants, 284. 
Trevirands (L.-C.). Nouv, observations sur l'avortement de la corolle et sur l'influence qu'il exerce, 178 .

Tulasne (L.-R.). Note sur les Isaria et Spharia entomogènes, 182.

Turczanisow (N.). Animadversiones in secundam partem herbarii Turczaninowiani, nunc universitatis casarecs charkowiensis, 770.

VAn-Hatl (H.). Observationes de Zingiberaceis, 139.

Weddell (H.-A.). Chloris andina (t. I), 383.

WeIss (Ad.). Note pour servir à l'histoire des stomates, 123. - Sur une nouvelle situation des stomates et quelques autres remarques sur ces organes, 125.

Wright (C.), voy. Lindley.

Wulfen. Flora norica phanerogama (publ. par MM. Fenzl et Graf), 231.

Zanardini (G.). Catalogue des plantes cryptogames récoltées jusqu'à ce jour dans les provinces vénitiennes ( ${ }^{\text {re }}$ série : Phycées), 780.

Zetterstedt (J.-E.). Plantes vasculaires des Pyrénées principales, 61.

Zullinger (H.). Sur un nouv. genre de Palmiers (Teysmania) de l'lle de Sumatra, 185. - Voy. Choisy. 


\section{$2 \mathrm{BHL}$ Biodiversity Heritage Library}

1858. "Table Par Ordre Alphabétique Des Noms D'Auteur Des Publications Analysées Dans La Revue Bibliographique." Bulletin de la Société botanique de France 5, 799-802. https://doi.org/10.1080/00378941.1858.10829335.

View This Item Online: https://www.biodiversitylibrary.org/item/8629

DOI: https://doi.org/10.1080/00378941.1858.10829335

Permalink: https://www.biodiversitylibrary.org/partpdf/158162

\section{Holding Institution}

Missouri Botanical Garden, Peter H. Raven Library

\section{Sponsored by}

Missouri Botanical Garden

\section{Copyright \& Reuse}

Copyright Status: Public domain. The BHL considers that this work is no longer under copyright protection.

This document was created from content at the Biodiversity Heritage Library, the world's largest open access digital library for biodiversity literature and archives. Visit BHL at https://www.biodiversitylibrary.org. 